\title{
ON ITERATES OF CONVOLUTIONS
}

\author{
SHAUL R. FOGUEL ${ }^{1}$
}

ABSTRACT. Let $\mu$ be a signed measure, of total variation one, on a locally compact Abelian group. We study in this note the ideal $I=$ $\left\{\tau: \tau \ll m\right.$ and $\left.\left\|\mu^{n} * \tau\right\|-0\right\}$ where $m$ is the Haar measure.

Let $G$ be a locally compact Abelian group, $\Gamma$ its dual group and $m$ its Haar measure. Throughout this note we shall use the notation of [5].

Let $\mu$ be a fixed signed measure (not necessarily continuous with respect to $m$ ), let $\nu$ be the total variation of $\mu$. We shall assume throughout:

Assumption, $\nu(G)=1$.

Definition 1. $I=\left\{\tau: \tau \ll m\right.$ and $\left\|\mu^{n} * \tau\right\| \rightarrow 0$ as $\left.n \rightarrow \infty\right\}$.

Definition 2. Let $J$ be the closed ideal, of signed measures, generated by $\left(\mu_{A}-\nu(A) \mu\right) * \eta$ where $\eta \ll m, A$ is a mea surable subset of $G$ and $\mu_{A}$ is the restriction of $\mu$ to $A$.

Lemma 1. $J \subset I$.

Proof. Let $A \subset G$ satisfy $0<\nu(A)<1$. Put $\mu_{1}=\nu(A)^{-1} \mu_{A}, \mu_{2}=$ $(1-\nu(A))^{-1}\left(\mu-\mu_{A}\right)$. Then $\mu=\nu(A) \mu_{1}+(1-\nu(A)) \mu_{2}$ and, by Lemma 2.1 of [3], $\left\|\mu^{n} *\left(\mu_{1}-\mu_{2}\right)\right\| \rightarrow 0$. Now

$$
\mu_{1}-\mu_{2}=\nu(A)^{-1}(1-\nu(A))^{-1}\left(\mu_{A}-\nu(A) \mu\right),
$$

hence $\left\|\mu^{n} *\left(\mu_{A}-\nu(A) \mu\right)\right\| \rightarrow 0$. Clearly this remains true if $\nu(A)=0$ or $\nu(A)=1$ too. Thus, $\left(\mu_{A}-\nu(A) \mu\right) * \eta \in I$ whenever $\eta \ll m$.

Theorem 1. Let $\gamma \in \Gamma$; the following conditions are equivalent:

(a) $\gamma$ vanishes on $\mathrm{J}$.

(b) $(d \mu / d \nu)(x)=(x, \gamma) \hat{\mu}(\gamma)$ a.e. $\nu$.

(c) $|\hat{\mu}(\gamma)|=1$.

(d) $\gamma$ vanishes on $l$.

Received by the editors August 2, 1973 and, in revised form, November 20, 1973. AMS (MOS) subject classifications (1970). Primary 43A25, 60J 15.

1 The research reported in this note was supported in part by NSF grant GP28933. 
Proof. $(\mathrm{a}) \Rightarrow$ (b). If $\left(\hat{\mu}_{A}(\gamma)-\nu(A) \hat{\mu}(\gamma)\right) \cdot \hat{\eta}(\gamma)=0$ for every $\eta \ll m$ and every set $A$, then $\int_{A}(-x, \gamma) \mu(d x)=\int_{A} \hat{\mu}(\gamma) \nu(d x)$. Now $\int_{A}(-x, \gamma) \mu(d x)=$ $\int_{A}(-x, \gamma)(d \mu / d \nu)(x) \nu(d x)$ from which (b) follows.

(b) $\Rightarrow$ (c). Note that $|(x, \gamma)|=1$ and $|(d \mu / d \nu)(x)|=1$ a.e. $\nu$.

(c) $\Rightarrow$ (d). If $\left\|\mu^{n} * \tau\right\| \rightarrow 0$ where $|\hat{\mu}(\gamma)|=1$, then $|\hat{\tau}(\gamma)|=\left|\widehat{\mu^{n} * \tau(\gamma)}\right|=0$.

(d) $\Rightarrow$ (a). Follows from Lemma 1 .

Definition 3. $E=E(\mu)=\{\gamma: \gamma \in \Gamma$ and $\hat{\tau}(\gamma)=0$ for every $\tau \in I\}=$ $\{\gamma: \gamma \in \Gamma$ and $\hat{\tau}(\gamma)=0$ for every $\tau \in J\}$.

Let us use now the notion of a set of spectral synthesis, see $[5,7.1 .4$, p. 158]. Note that by (c) of Theorem $1, E$ is a closed set. Now if $\gamma_{0} \in E$ and $\gamma_{0}+\gamma \in E$ it follows from (b) of Theorem 1 that

$$
\left(x, \gamma_{0}\right) \hat{\mu}\left(\gamma_{0}\right)=\left(x, y+\gamma_{0}\right) \hat{\mu}\left(y+\gamma_{0}\right) \text { a.e. } \nu
$$

hence $(x, y)=$ const. a.e. $\nu$. Conversely, if $\gamma_{0} \in E$ and $(x, \gamma)=$ const. a.e. $\nu$ then

$$
\hat{\mu}\left(\gamma_{0}+\gamma\right)=\int\left(-x, \gamma_{0}\right)(-x, \gamma) \mu(d x)=\text { const. } \hat{\mu}\left(\gamma_{0}\right)
$$

and $\gamma_{0}+\gamma \in E$ too.

Thus $E$ is a coset of $\Gamma$ and by $[5,7.5 .2$, part (d), p. 170] $E$ is a set of spectral synthesis. Thus

Theorem 2. $I=J=\{r: \tau \ll m$ and $\hat{\gamma}(\gamma)=0$ for all $\gamma \in E\}$.

Remark. From part (b) of Theorem 1 follows that if $d \mu / d \nu$ is not a multiple of $(x, \gamma)$, for some $\gamma$, a.e. $\nu$ then $J=I=L_{1}(G)$.

Theorem 3. If $\nu$ is not supported on a set of the form $\left\{x:\left(x, \gamma_{0}\right)=\right.$ const. $\}$ for some $\gamma_{0} \in \Gamma$, then $E$ is either empty or contains one point.

Proof. Let $\gamma_{1}, \gamma_{2} \in E$; then $\left(x, \gamma_{1}\right)=\hat{\mu}\left(\gamma_{1}\right)$ and $\left(x, \gamma_{2}\right)=\hat{\mu}\left(\gamma_{2}\right)$ a.e. $\nu$. Hence $\left(x, \gamma_{1}-\gamma_{2}\right)=$ const. a.e. $\nu$ which contradicts our assumption.

Remark. Assume the contradiction of Theorem 3 and $d \mu(x) / d \nu=c(x, \gamma)$ a.e. $\nu$. Then $\hat{\mu}(\gamma)=c$ and $\gamma \in E$.

Corollary l. If $\mu \geq 0$ and the support of $\mu$ is not contained in any set of the form $\left\{x:\left(x, \gamma_{0}\right)=\right.$ const. $\}$, then $\left\|\mu^{n} * \tau\right\| \rightarrow 0$, as $n \rightarrow \infty$, whenever $\tau \ll m$ and $\tau(G)=0$.

Proof. If $\mu \geq 0$ then $0 \in E$ ( 0 is the character which is identically equal to 1). Thus $E=\{0\}$ by Theorem 3 . 
Remark. For $G=R^{1}$ this corollary was proved in [4, The orem 7, part (b), p. 11], by a probabilistic argument.

The following corollary was suggested to us by John Baxter.

Corollary 2. If the support of $\mu$ is not contained in any set of the form $\{x:(x, y)=$ const. $\}$ and $f$ is a measurable bounded function such that $\mu * f=f$ a.e. $m$, then $f=$ const. a.e. $m$.

Proof. By Corollary 1 if $\tau \ll m$ and $\tau(G)=0$, then

$$
|\tau * f|=\left|\left(\tau * \mu^{n}\right) * f\right| \leq\|f\|_{\infty}\left\|\tau * \mu^{n}\right\|_{1} \rightarrow 0 .
$$

Thus $f=$ const. a.e. $m$.

Remarks. Corollary 2 is proved in [2, Chapter XI, \$2, p. 351]. It is proved also in [1] for non-Abelian groups but under the restrictive assumption that $\mu$ induces a conservative random walk. Also in [1] it is shown that this corollary implies that the only bounded measure $\tau$, with $\tau * \mu=\tau$ is a multiple of Haar measure if $G$ is compact and is zero otherwise. Finally note that Corollary 2 is a generalization of the classical result that a bounded harmonic function is a constant: take $G=R^{n}$ and $\mu$ a uniform dis. tribution on a sphere around the origin.

\section{BIBLIOGR APHY}

1. A. Brunel, P. Crepel, Y. Guivarc'h and M. Keane, Marches aléatoires recurrentes sur les groupes localement compacts, C. R. Acad. Sci. Paris 275 (1972), 1359-1361.

2. W. Feller, $A_{n}$ introduction to probability theory and its applications. Vol. II, Wiley, New York, 1966. MR $35 \# 1048$.

3. S. R. Foguel and B. Weiss, On convex power series of conservative Markov operator, Proc. Amer. Math. Soc. 38 (1973), 325-330.

4. D. S. Ornstein, Random walks. I, Trans. Amer. Math. Soc. 138 (1969), 1-43. MR $38 \# 6675$.

5. W. Rudin, Fourier analysis on groups, Interscience Tracts in Pure and Appl. Math., no. 12, Interscience, New York, 1962. MR 27 \#2808.

DEPARTMENT OF MATHEMATICS, UNIVERSITY OF MINNESOTA, MINNEAPOLIS, MINNESOT A 55455

DEPARTMENT OF MATHEMATICS, HEBREW UNIVERSITY OF JERUSAL EM, JERUSALEM, ISRAEL 